\title{
UN ALGORITHME D'IDENTIFICATION DE FRONTIÈRES SOUMISES À DES CONDITIONS AUX LIMITES DE SIGNORINI
}

\author{
${\text { Slim } \text { ChaAbane }^{1} \text { And Mohamed JaOuA }}^{2}$
}

\begin{abstract}
This work deals with a non linear inverse problem of reconstructing an unknown boundary $\gamma$, the boundary conditions prescribed on $\gamma$ being of Signorini type, by using boundary measurements. The problem is turned into an optimal shape design one, by constructing a Kohn \& Vogelius-like cost function, the only minimum of which is proved to be the unknown boundary. Furthermore, we prove that the derivative of this cost function with respect to a direction $\theta$ depends only on the state $u^{0}$, and not on its Lagrangian derivative $u^{1}(\theta)$.

Résumé. On s'intéresse dans ce travail à un problème inverse non linéaire d'identification d'une frontière inconnue $\gamma$ par des mesures de surfaces, les conditions aux limites imposées sur cette frontière $\gamma$ étant de type Signorini. Le problème est d'abord transformé en un problème d'optimisation de forme, par la définition d'une fonction de type Kohn-Vogelius, dont nous montrons que le seul minimum est la frontière recherchée, et que le gradient dans une direction donnée $\theta$ ne dépend que du seul état $u^{0}$, et non de sa dérivée lagrangienne $u^{1}(\theta)$.
\end{abstract}

Mathematics Subject Classification. 35R30, 35S85, 49Q10, 49Q12, 49M10, 65K10.

Reçu : 29 juin 1998. Révisé : 14 janvier 2000.

\section{Extended English Abstract}

We are interested in this work in the study of a geometrical inverse problem, which consists in finding the shape of an unknown part $\gamma$ of the boundary $\partial \Omega$ of a two-dimensional body $\Omega$, by using thermal measurements on some part $M$ of the boundary. The two extremal points of the unknown boundary $\gamma$ are supposed to be fixed, while Signorini type boundary conditions are prescribed on $\gamma$.

Most the numerical algorithms developed for identification purposes are based on a least squares approach: a cost function, to be minimized, is defined as the quadratic gap between the measured data, and the corresponding response computed by solving - using the prescribed flux $\phi$ - a Neumann boundary value problem on the domain. The unknown part of the boundary is then updated. Kohn \& Vogelius [10] improved this approach by proposing a "variational" cost function, representing the energy gap between the "Neumann" solution $u_{N}$, and the "Dirichlet" one $u_{D}$ computed using the measured data $f$. By setting the boundary conditions in a right way, one can get an expression of the cost function as a sum of two uncoupled compliance functions, the derivatives of which - with respect toi the domain - turn out not to depend on the domain derivatives of $u_{N}$

Keywords and phrases. Geometrical inverse problems, identification, Signorini type boundary conditions, unknown boundary, domaine derivatives, Kohn-Vogelius function, optimal shape design.

1 Faculté des Sciences de Sfax \& ENIT-LAMSIN. e-mail: slim.chaabane@fsm.rnu.tn

2 ENIT-LAMSIN, BP 371002 Tunis-Belvédère, Tunisie. e-mail: mohamed.jaoua@enit.rnu.tn 
and $u_{D}$. Such a result is of great interest if one has in mind to use a gradient algorithm to minimize this cost function. This ide a was first numerically tested in [8]. It generalizes quite well to several other situations, and many authors have made use of it to implement numerical algorithms.

In non linear cases however, both features described above are lost. A coupling term remains in the cost function, generating the presence of the derivatives of $u_{D}$ and $u_{N}$ in its own derivative. The reason is that there are actually two different partitions of the unknown boundary $\gamma$ into a "Dirichlet part" $\gamma_{D}$ where the Dirichlet condition $u=0$ holds, and a "Neumann part $\gamma_{N}$, where the Neumann condition $\frac{\partial u}{\partial n}=0$ is fulfilled. The first partition is related to $u_{N}$, and the second one to $u_{D}$.

It seems therefore natural to force the partition in the associated Dirichlet problem, by replacing its Signorini boundary condition on $\gamma$ by a mixed Neumann-Dirichlet one, using for that the partition derived from the Neumann solution $u_{N}$. Such a trick is actuially possible because the Neumann-Signorini solution $u_{N}$ is smooth enough [7], so that $\gamma_{N}$ is an open subset of $\gamma$, and $\gamma_{D}$ a closed one, and the mixed boundary conditions on $\gamma_{N}$ and $\gamma_{D}$ make sense.

This idea works quite well, although the two parts of the cost function remain weakly coupled by the way of the $\gamma$-partition. We first prove that the this cost function, defined by:

$$
J(\gamma)=\int_{\Omega_{\gamma}}\left|\nabla u_{N}^{0}-\nabla u_{D}^{0}\right|^{2}
$$

has a unique minimum, which is the unknown boundary. In order to compute the derivative of the cost function with respect to the domain, we use a "Lagrangian" representation, that is to consider perturbated domains $\Omega_{h}$ which are images by a mapping $I d+h \theta$ of the original domain. $\theta$ is the direction of perturbation, and we define the derivative as:

$$
\frac{\partial J}{\partial \Omega}(\Omega) \cdot \theta=\lim _{h \rightarrow 0} \frac{J\left(\gamma_{h}\right)-J(\gamma)}{h}
$$

Both Dirichlet and Neumann solutions on the perturbated domain, transported to the original domain $\Omega$ by $u^{h}:=u_{h} \circ(I d+h \theta)$, expand in the energy space with respect to the parameter $h[16]$ :

$$
u_{D}^{h}=u_{D}^{0}+h u_{D}^{1}+h o_{1}(h)
$$

and :

$$
u_{N}^{h}=u_{N}^{0}+h u_{N}^{1}+h o_{2}(h)
$$

with $\lim _{h \rightarrow 0} o_{i}(h)=0 ; i=1,2 . u_{N}^{1}$ and $u_{D}^{1}$ are actually the domain derivatives of the solutions with respect to the direction $\theta . u_{N}^{h}$ defines a new partition of the Signorini boundary $\gamma$ in $\gamma_{N}^{h}$ and $\gamma_{D}^{h}$. To prove that the derivative of the cost function does not actually depend on $u_{N}^{1}$ and $u_{D}^{1}$, we need first to prove a continuity result on this partition, namely:

$$
\lim _{h \rightarrow 0} d\left(\gamma_{N}^{h}, \gamma_{N}\right)=0
$$

This result is obtained under the assumption that the number of connected components in $\gamma_{N}^{h}$ is bounded 
independently of $h$. The expressions of the domain derivatives are therefore the following:

$$
\lim _{h \rightarrow 0^{+}} \frac{J\left(\gamma_{h}\right)-J(\gamma)}{h}=2 \int_{\Omega}\left(\left\langle D \theta \nabla u_{N}^{0}, \nabla u_{N}^{0}\right\rangle-\left\langle D \theta \nabla u_{D}^{0}, \nabla u_{D}^{0}\right\rangle\right)-\int_{\Omega_{\gamma}} \operatorname{div} \theta\left(\left|\nabla u_{N}^{0}\right|^{2}-\left|\nabla u_{D}^{0}\right|^{2}\right)
$$

If $u_{N}^{0} \in H^{2}(\operatorname{supp}(\theta))$, then we can get a boundary integral expression of this derivative:

$$
\left.\left.\lim _{h \rightarrow 0^{+}} \frac{J\left(\gamma_{h}\right)-J(\gamma)}{h}=\int_{\gamma_{D}}\left[\left(\frac{\partial u_{N}^{0}}{\partial n}\right)^{2}-\left(\frac{\partial u_{D}^{0}}{\partial n}\right)^{2}\right)\right] \theta_{n}+\int_{\gamma_{N}}\left[\left(\frac{\partial u_{D}^{0}}{\partial \tau}\right)^{2}-\left(\frac{\partial u_{N}^{0}}{\partial \tau}\right)^{2}\right)\right] \theta_{n}
$$

These expressions allow us to implement a gradient algorithm to solve our inverse problem. The first numerical results obtained are quite good, the computing time being dramatically reduced by the linearization (from Signorini to mixed boundary conditions on the auxiliary Dirichlet problem), and by the use of the above gradient expressions, which do not depend on the derivatives of the solution. To us however, the main interest of this work lie in the way used to overcome the difficulties arising from the non linear boundary conditions. It appears that this approach may be extended to the thermo elastic coupled problem, which is a more relevant problem from a physical point of view.

\section{IntroduCtion}

Soit $\Omega$ un ouvert borné de classe $\mathcal{C}^{2}$ de $\mathbb{R}^{2}$, de frontière $\partial \Omega=\gamma \cup \Gamma_{D} \cup \Gamma_{N}, \gamma$ désignant la partie inconnue de la frontière $\partial \Omega$.

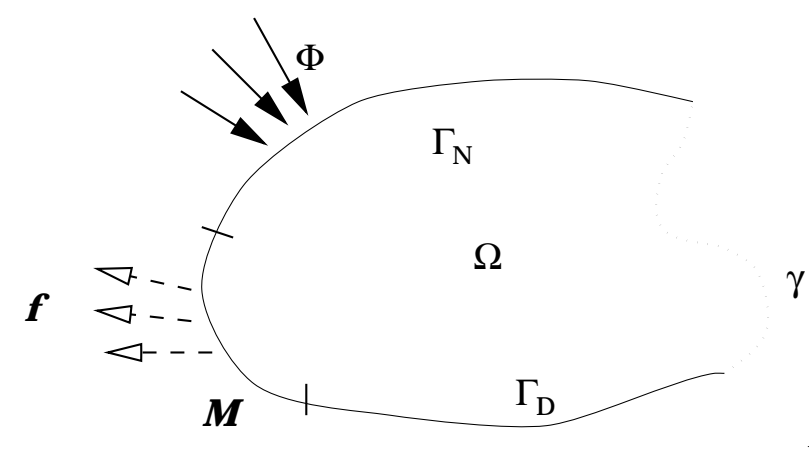

Figure 1. Le domaine et sa frontière.

Considérons le problème de Signorini :

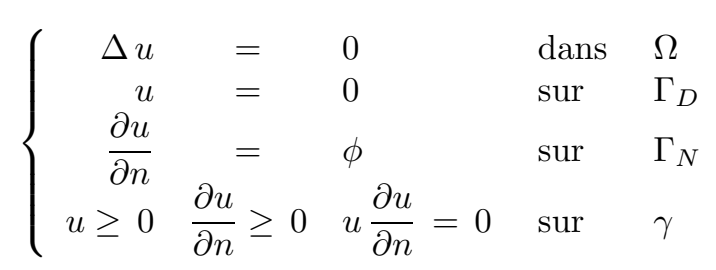

où $\phi$ désigne le flux de chaleur imposé sur $\Gamma_{N}$, que nous supposerons non nul :

$$
\phi \not \equiv 0 \operatorname{sur} \Gamma_{N} .
$$


L'inconnue du problème est la frontière $\gamma$, que l'on s'efforcera de déterminer au moyen de mesures effectuées sur une partie $M$ de $\Gamma_{N}$, de mesure positive. Désignant par $\Gamma_{a d}$ l'ensemble des frontières $\gamma$ admissibles, partie d'une courbe de Jordan de classe $\mathcal{C}^{1, \beta} ; \beta>0$ ayant leurs deux extrémités fixés, le problème d'identification peut être formulé comme suit :

$$
\left\{\begin{array}{l}
\text { Trouver } \gamma \in \Gamma_{a d} \text { tel que } \\
u_{\gamma}, \text { solution de (8), vérifie }\left.u_{\gamma}\right|_{M}=f .
\end{array}\right.
$$

Dans un précédent travail [2], il a été montré que sous les hypothèses de régularité énoncées ci-dessus, une seule mesure de frontière $\left(u_{\gamma}=f \in \mathrm{H}^{\frac{1}{2}}(M)\right)$ suffit pour déterminer la frontière inconnue $\gamma$ de manière unique (résultat d'identifiabilité). Dans ce même travail, nous avons aussi montré un résultat de stabilité locale lipshitzienne, signifiant une dépendance continue de la frontière identifiée par rapport à la mesure effectuée, ce qui permet d'envisager la mise en œuvre d'algorithmes numériques d'identification.

Ces derniers sont le plus souvent basés sur une approche de type «moindres carrés» : on définit une fonction coût mesurant l'écart quadratique entre les températures mesurées et celles que l'on calcule en utilisant le flux imposé, pour une réalisation donnée de la frontière inconnue $\gamma$, que l'on s'efforce ensuite de minimiser par un algorithme approprié, la frontière étant ajustée à chaque itération. Ce choix n'est évidement pas le seul possible, Kohn et Vogelius [9,10] en ayant d'ailleurs proposé un meilleur, basé sur l'écart énergétique, pour une réalisation donnée $\gamma$ de la frontière inconnue, entre la solution du problème de Neumann défini en utilisant le flux imposé comme condition aux limites, et celle du problème aux limites de Dirichlet défini en tirant parti de la donnée mesurée sur $M$. Dans les cas linéaires, il apparaît que cette fonctionnelle est dérivable par rapport au domaine, et que sa dérivée lagrangienne ne dépend que de l'état, et non de la dérivée lagrangienne de celui-ci. Cette approche, initiée de longue date [15], se révèle en outre apte à la généralisation, ainsi qu'à des développements numériques, comme l'ont illustré plusieurs travaux $[5,6,8,11,14] \ldots$

Les conditions aux limites non linéaires soulèvent toutefois des difficultés particulières : la fonction coût de Kohn et Vogelius ne peut plus s'exprimer comme somme de deux compliances découplées (l'une relative au problème de Neumann, et l'autre au problème de Dirichlet), de sorte que sa dérivée lagrangienne ne peut s'exprimer indépendamment de celles des états. Ce couplage est imputable au fait que les deux partitions de la frontière inconnue $\gamma$ (entre une partie où la solution $u$ s'annule, et une autre où c'est sa dérivée normale qui s'annule) ne sont pas identiques pour la solution de Dirichlet et celle de Neumann. Il parait dès lors naturel d'y remédier en forçant la partition de la frontière $\gamma$ pour le problème auxiliaire, ce qui en constitue une linéarisation (on résout un problème mêlé au lieu d'un problème de Signorini), et a donc pour effet annexe de réduire considérablement les coûts de calcul dans l'algorithme subséquent. La fonctionnelle ainsi définie s'écrit alors comme la somme de deux termes faiblement couplés, sa dérivée lagrangienne ne dépendant cependant que des seuls états, et ce grâce à un résultat de continuité de la partition Dirichlet-Neumann de la frontière inconnue par rapport au domaine.

Dans la suite on désignera par $\Omega_{\gamma}$ le domaine $\Omega$ de frontière inconnue $\gamma$, l'état $u$ correspondant étant noté $u_{\gamma}$.

\section{Fonctionnelles de Kohn et Vogelius}

Dans la suite, on suppose que l'on connait la température $f$ sur tout le support de $\phi$, et on désigne par $u_{N}^{0}$ la solution de (8), et par $u_{D S}^{0}$ la solution du problème de Dirichlet-Signorini suivant :

$$
\left\{\begin{array}{cclll}
\Delta u_{D S}^{0} & = & 0 & \text { dans } & \Omega_{\gamma} \\
u_{D S}^{0} & = & 0 & \text { sur } & \Gamma_{D} \\
u_{D S}^{0} & = & f & \text { sur } & \operatorname{supp}(\phi) \\
\frac{\partial u_{D S}^{0}}{\partial n} & = & 0 & \text { sur } & \Gamma_{N} \backslash \operatorname{supp}(\phi) \\
u_{D S}^{0} \geq 0 & \frac{\partial u_{D S}^{0}}{\partial n} \geq 0 & u_{D S}^{0} \frac{\partial u_{D S}^{0}}{\partial n}=0 & \text { sur } & \gamma
\end{array}\right.
$$


La fonctionnelle de Kohn-Vogelius associée à ce problème inverse est alors la suivante :

$$
K(\gamma)=\int_{\Omega_{\gamma}}\left|\nabla u_{N}^{0}-\nabla u_{D S}^{0}\right|^{2}
$$

soit encore :

$$
K(\gamma)=\int_{\Omega_{\gamma}}\left|\nabla u_{N}^{0}\right|^{2}+\int_{\Omega_{\gamma}}\left|\nabla u_{D S}^{0}\right|^{2}-2 \int_{\Omega_{\gamma}}\left\langle\nabla u_{N}^{0}, \nabla u_{D S}^{0}\right\rangle
$$

Cette fonctionnelle contenant un terme de couplage $\int_{\Omega_{\gamma}}\left\langle\nabla u_{N}^{0}, \nabla u_{D S}^{0}\right\rangle$, sa dérivée par rapport aux domaines dans une direction $\theta$ dépend des dérivées lagrangiennes $u_{N}^{1}(\theta)$ et $u_{D S}^{1}(\theta)$ des états $u_{N}^{0}$ et $u_{D S}^{0}$, ce qui conduit à des algorithmes lourds à mettre en œuvre numériquement. On s'intéresse dans la suite à modifier cette fonctionnelle de manière à obtenir une nouvelle fonctionnelle ayant le même point critique, mais dont la dérivée par rapport au domaine s'exprime indépendamment des dérivées lagrangiennes des états.

\subsection{Construction de la fonctionnelle $J$}

La résolution du problème (8) nous permet de définir les surfaces de contacts $\gamma_{D}$ et $\gamma_{N}$ par :

$$
\gamma_{D}=\left\{x \in \gamma ; u_{N}^{0}(x)=0\right\} \quad ; \quad \gamma_{N}=\left\{x \in \gamma ; \in u_{N}^{0}(x)>0\right\}
$$

Désignons par $u_{D}^{0}$ la solution du problème :



On définit alors la fonction coût $J$ par :

$$
J(\gamma)=\int_{\Omega_{\gamma}}\left|\nabla u_{N}^{0}-\nabla u_{D}^{0}\right|^{2}
$$

Théorème 2.1. Il existe une et une seule frontière $\gamma \in \Gamma_{a d}$ telle que :

$$
J(\gamma) \leq J\left(\gamma^{\prime}\right) \quad \forall \gamma^{\prime} \in \Gamma_{a d}
$$

De plus, $\gamma$ est la solution du problème inverse (9).

Preuve. Il est clair que pour $\gamma$ solution du problème inverse $u_{N}^{0}=u_{D}^{0}$, donc $\gamma$ est un minimum pour $J$ avec $J(\gamma)=0$. Soit $\gamma_{1}$ un autre minimum de $J, J\left(\gamma_{1}\right)=0$, donc $u_{N}^{0}=u_{D}^{0}$. Il en résulte que, pour cette frontière $\gamma_{1}, u_{N}^{0}$ vérifie le problème (8) avec $u_{N}^{0}=f$ sur $M$. Le résultat d'identifiabilité ( $c f$. [2]) nous donne alors que $\gamma_{1}=\gamma$ 


\section{DÉRIVATiOn De LA FOnCTION COÛT}

\subsection{Dérivation lagrangienne}

Soit $\theta$ un champ de vecteurs de classe $\mathcal{C}^{2}$ dans un voisinage de $\Omega_{\gamma}$, nul dans un voisinage de $\Gamma_{D} \cup \Gamma_{N}$, non identiquement nul sur $\gamma$ et porté par la normale sur $\gamma$. Pour $h>0$ assez petit, le champs $I d+h \theta$ constituera une cinématique virtuelle dans la direction $\theta$.

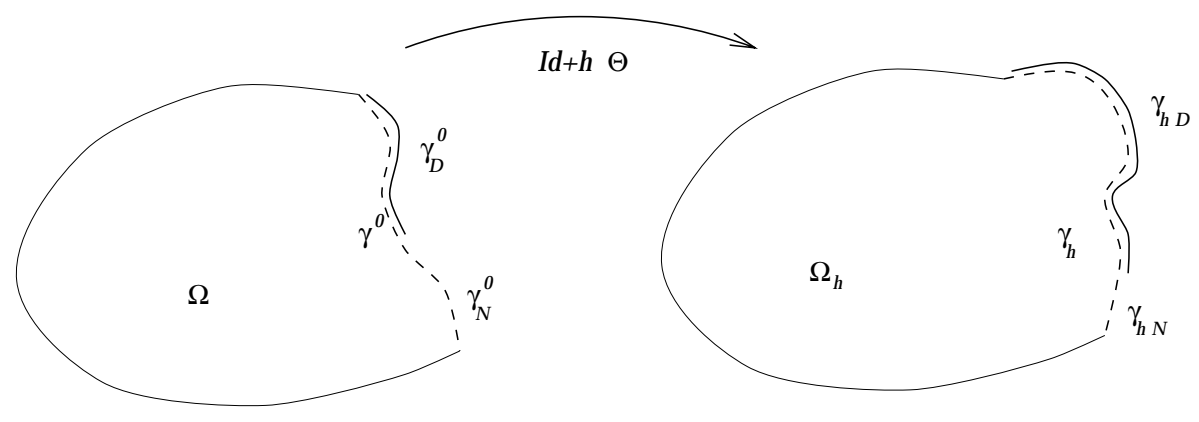

Figure 2. Perturbation du domaine $\Omega$.

Désignons par $\Omega_{h}=(I d+h \theta)\left(\Omega_{\gamma}\right)$, et par $u_{h N}$ (resp. $u_{h D}$ ) la solution du problème (8) (resp. la solution du problème (13)) posé dans $\Omega_{h}$ et par $u_{N}^{h}\left(\right.$ resp. $\left.u_{D}^{h}\right)$ son transporté sur le domaine d'origine $\Omega_{\gamma}$

$$
u_{N}^{h}=u_{h N} \circ(I d+h \theta)
$$

et

$$
u_{D}^{h}=u_{h D} \circ(I d+h \theta) .
$$

Désignons par $u_{N}^{1}\left(\operatorname{resp} u_{D}^{1}\right)$ les dérivées lagrangiennes de $u_{N}^{0}$ (resp de $\left.u_{D}^{0}\right)$ et par $\gamma_{h}=(I d+h \theta)(\gamma)$.

\subsection{Continuité de la partition de $\gamma$ par rapport au domaine}

Définissons à présent $\gamma_{D}^{h}=\left\{x \in \gamma ; u_{N}^{h}(x)=0\right\}$ et $\gamma_{N}^{h}=\left\{x \in \gamma ; u_{N}^{h}(x)>0\right.$.

Lemme 3.1. Désignant par $\mu$ la mesure de Lebesgue sur $\gamma$, on a :

$$
\lim _{h \rightarrow 0^{+}} \mu\left(\gamma_{D}^{h} \backslash \gamma_{D}\right)=0
$$

Preuve. Le développement limité du premier ordre de $u_{N}^{h}(c f .[16])$, nous donne, pour une constante $c>0$, la majoration suivante :

$$
\left|u_{N}^{h}-u_{N}^{0}\right|_{L^{1}(\gamma)} \leq c h
$$

ce qui, puisque $u_{N}^{h} \equiv 0$ sur $\gamma_{D}^{h}$, nous donne :

$$
\int_{\left(\gamma_{D}^{h} \backslash \gamma_{D}\right)}\left|u_{N}^{0}\right|=\int_{\left(\gamma_{D}^{h} \backslash \gamma_{D}\right)}\left|u_{N}^{h}-u_{N}^{0}\right| \leq c h .
$$

Puisque $u_{N}^{0}>0 \operatorname{sur}\left(\gamma_{D}^{h} \backslash \gamma_{D}\right)$, on peut déduire que $\lim _{h \rightarrow 0^{+}} \mu\left(\gamma_{D}^{h} \backslash \gamma_{D}\right)=0$. En effet, désignant par $\chi_{h}$ la fonction caractéristique de l'ensemble $\gamma_{D}^{h} \backslash \gamma_{D}$, nous obtenons :

$$
\lim _{h \rightarrow 0} \int_{\left(\gamma_{D}^{h} \backslash \gamma_{D}\right)}\left|u_{N}^{0}\right|=\lim _{h \rightarrow 0} \int_{\gamma_{N}} \chi_{h}\left|u_{N}^{0}\right|=0
$$


ce qui signifie que la fonction positive $\chi_{h}\left|u_{N}^{0}\right|$ tend vers zéro dans $\mathrm{L}^{1}\left(\gamma_{N}\right)$, donc presque partout sur $\gamma_{N}$, et qu'en conséquence $\chi_{h}$ tend vers zéro presque partout sur $\gamma_{N}$ puisque $\left|u_{N}^{0}\right|$ est strictement positive sur cet ensemble. Il en résulte que $\lim _{h \rightarrow 0^{+}} \mu\left(\gamma_{D}^{h} \backslash \gamma_{D}\right)=0$.

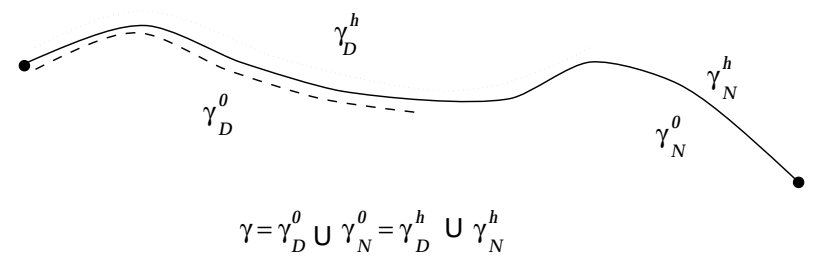

Figure 3. Les deux partitions de la frontière $\gamma$.

Il n'est toutefois pas possible d'étendre ce raisonnement à la dérivée normale, pour obtenir un résultat similaire sur $\left(\gamma_{N}^{h} \backslash \gamma_{N}\right)$, et achever ainsi la preuve de la continuité de la partition. La raison est en double :

- D'une part, $\gamma_{N}$ ne peut être caractérisé (même à un ensemble négligeable près) comme la partie de $\gamma$ sur laquelle $\frac{\partial u_{N}^{0}}{\partial n}$ s'annule. Dans [2], nous montrons en fait que :

$$
\stackrel{\circ}{\gamma_{D}}=\left\{x \in \gamma ; \frac{\partial u_{N}^{0}}{\partial n}>0\right\} .
$$

- D'autre part, le transport de $\gamma_{h}$ sur $\gamma$ ne conserve pas la dérivée normale, mais plutôt la dérivée co-normale de $u_{N}^{h}$, à la quelle nous nous proposons maintenant de nous intéresser.

\subsubsection{Continuité de la dérivée co-normale}

Commençons par rappeler le problème variationnel vérifié par $u_{N}^{h}$. Notons par $F_{h}:=I d+h \theta, M_{h}:=$ $\left(D F_{h}\right)^{-1}, u_{N}^{h}$ est alors [16] solution du problème variationnel suivant :

$$
\left\{\begin{array}{l}
\text { Trouver } u \in K \text { telle que } \\
a^{h}(u, v-u) \geq \int_{\Gamma_{N}} \phi(v-u) \forall v \in K
\end{array}\right.
$$

où $K$ désigne le convexe fermé de $\mathrm{H}^{1}\left(\Omega_{\gamma}\right)$ défini par :

$$
K=\left\{u \in \mathrm{H}^{1}\left(\Omega_{\gamma}\right) ; u \mid \Gamma_{D}=0 ; u \geq 0 \text { p.p. sur } \gamma\right\}
$$

et $a^{h}$ la forme bilinéaire définie sur $\mathrm{H}^{1}\left(\Omega_{\gamma}\right)$ par :

$$
a^{h}(u, v)=\int_{\Omega_{\gamma}} \operatorname{det}\left(D F_{h}\right)\left\langle\left(M_{h}{ }^{t} M_{h}\right) \nabla u, \nabla v\right\rangle .
$$

La forme bilinéaire $a^{h}$ est continue, coercive sur le sous-espace de Hilbert :

$$
V=\left\{v \in \mathrm{H}^{1}\left(\Omega_{\gamma}\right) ; v=0 \text { p.p sur } \Gamma_{D}\right\} .
$$

En prenant $\varphi \in \mathcal{D}\left(\Omega_{\gamma}\right)$ et en appliquant (19) à chacune des fonctions test $v_{1}=\varphi+u_{N}^{h}$ et $v_{2}=u_{N}^{h}-\varphi$ qui appartiennent toutes deux au convexe $K$, on déduit par intégration par parties que :

$$
\operatorname{div}\left[\operatorname{det}\left(D F_{h}\right)\left(M_{h}{ }^{t} M_{h}\right) \nabla u_{N}^{h}\right]=0 .
$$


Et par suite $u_{N}^{h}$ appartient à l'ensemble :

$$
\left\{u, u \in \mathrm{H}^{1}\left(\Omega_{\gamma}\right) ; \operatorname{div}\left[\operatorname{det}\left(D F_{h}\right)\left(M_{h}{ }^{t} M_{h}\right) \nabla u\right] \in \mathrm{L}^{2}\left(\Omega_{\gamma}\right)\right\} .
$$

On peut définir la dérivée co-normale (voir par exemple [12]) comme forme linéaire continue sur $\mathrm{H}_{00}^{\frac{1}{2}}(\gamma)$. Celui-ci est le sous-espace des fonctions de $\mathrm{H}^{\frac{1}{2}}(\gamma)$ qui se prolongent par 0 en fonctions de $\mathrm{H}^{\frac{1}{2}}(\Gamma)$, le prolongement étant continu. L'espace $\mathrm{H}_{00}^{\frac{1}{2}}(\gamma)$ est aussi l'interpolé d'ordre $\frac{1}{2}$ des espaces $\mathrm{H}_{0}^{1}(\gamma)$ et $\mathrm{L}^{2}(\gamma)$, et son dual topologique est par conséquent l'interpolé d'ordre $\frac{1}{2}$ des espaces duaux des deux précédents, à savoir $\mathrm{H}^{-1}(\gamma)$ et $\mathrm{L}^{2}(\gamma)$, cet interpolé n'est autre que l'espace $\mathrm{H}^{-\frac{1}{2}}(\gamma)$ (voir [13]). Ainsi :

1. Pour $\varphi \in \mathcal{D}(\gamma)$ et si $\tilde{\varphi}$ est un relèvement de $\varphi$ dans $\mathrm{H}^{1}\left(\Omega_{\gamma}\right)$ vérifiant $\tilde{\varphi}=0 \operatorname{sur}\left(\partial \Omega_{\gamma} \backslash \gamma\right)$

$$
\left\langle\frac{\partial u_{N}^{h}}{\partial \nu}, \varphi\right\rangle=a^{h}\left(u_{N}^{h}, \tilde{\varphi}\right)
$$

2. En prenant un relèvement continu $\mathcal{R}$ de $\mathrm{H}_{00}^{\frac{1}{2}}(\gamma)$ dans $\mathrm{H}^{1}\left(\Omega_{\gamma}\right)$, on définit $\frac{\partial u_{N}^{h}}{\partial \nu}$ de la même façon que plus haut, et on obtient pour une constante $c_{h}$ strictement positive la majoration suivante :

$$
\left|\left\langle\frac{\partial u_{N}^{h}}{\partial \nu}, \varphi\right\rangle\right| \leq c_{h}\|\mathcal{R}\|\|\varphi\|_{\frac{1}{2}, \gamma}
$$

Le crochet de dualité intervanant ci-dessus est celui de la dualité entre les espaces $\mathrm{H}^{-\frac{1}{2}}(\gamma)$ et $\mathrm{H}_{00}^{\frac{1}{2}}(\gamma)$

Lemme 3.2. Il existe $O(h) \in \mathrm{H}^{-\frac{1}{2}}(\gamma)$, borné indépendamment de $h$, tel que :

$$
\frac{\partial u_{N}^{h}}{\partial \nu}=\frac{\partial u_{N}^{0}}{\partial n}+h O(h) .
$$

Preuve. Soit $\varphi \in D(\gamma)$ et $\tilde{\varphi}$ un prolongement de $\varphi$ sur $\mathrm{H}^{1}\left(\Omega_{\gamma}\right) / \tilde{\varphi}=0 \operatorname{sur}\left(\partial \Omega_{\gamma} \backslash \gamma\right)$. Alors :

$$
\left\langle\frac{\partial u_{N}^{h}}{\partial \nu}-\frac{\partial u_{N}^{0}}{\partial n}, \varphi\right\rangle=\int_{\Omega_{\gamma}} \operatorname{det}\left(D F_{h}\right)\left\langle\left(M_{h}{ }^{t} M_{h}\right) \nabla u_{N}^{h}, \nabla \tilde{\varphi}\right\rangle-\int_{\Omega_{\gamma}}\left\langle\nabla u_{N}^{0}, \nabla \tilde{\varphi}\right\rangle .
$$

Nous avons :

avec :

$$
\operatorname{det}\left(D F_{h}\right)\left(M_{h}{ }^{t} M_{h}\right) \nabla u_{N}^{h}=\nabla u_{N}^{0}+h w+h o(h)
$$

et

$$
w=\operatorname{div} \theta \nabla u_{N}^{0}-\left(D \theta+{ }^{t} D \theta\right) \nabla u_{N}^{0}+\nabla u_{N}^{1}
$$

$$
\lim _{h \rightarrow 0^{+}} o(h)=0 \text { dans }\left(L^{2}\left(\Omega_{\gamma}\right)\right)^{2} .
$$

On a donc :

$$
\left|\left\langle\frac{\partial u_{N}^{h}}{\partial \nu}-\frac{\partial u_{N}^{0}}{\partial n}, \varphi\right\rangle\right|=h\left|\int_{\Omega_{\gamma}}\langle w, \nabla \tilde{\varphi}\rangle+\int_{\Omega_{\gamma}}\langle o(h), \nabla \tilde{\varphi}\rangle\right|
$$

Et on déduit l'existence d'une constante $c_{1}$ strictement positive telle que :

$$
\left|\left\langle\frac{\partial u_{N}^{h}}{\partial \nu}-\frac{\partial u_{N}^{0}}{\partial n}, \varphi\right\rangle\right| \leq c_{1} h\|\tilde{\varphi}\|_{1, \Omega_{\gamma}}
$$

En choisissant $\tilde{\varphi}=\mathcal{R}(\varphi)$, on obtient pour une constante $c>0$ indépendante de $\varphi$ et de $h$ la majoration suivante : 


$$
\left|\left\langle\frac{\partial u_{N}^{h}}{\partial \nu}-\frac{\partial u_{N}^{0}}{\partial n}, \varphi\right\rangle\right| \leq \operatorname{ch}\|\varphi\|_{\frac{1}{2}, \gamma}
$$

\subsubsection{Continuité de la partition de $\gamma$}

Commençons par établir le lemme suivant :

Lemme 3.3. $\frac{\partial u_{N}^{h}}{\partial \nu}=0$ sur $\gamma_{N}^{h}$.

Preuve. Soit $\varphi \in D(\gamma)$ tel que $\operatorname{supp}(\varphi) \subset \gamma_{N}^{h}$, et soit $\tilde{\varphi}=\mathcal{R}(\varphi)$.

On a :

$$
\left\langle\frac{\partial u_{N}^{h}}{\partial \nu}, \varphi\right\rangle=a^{h}\left(u_{N}^{h}, \tilde{\varphi}\right)
$$

Or :

$$
a^{h}\left(u_{N}^{h}, \tilde{\varphi}\right)=\int_{\Omega_{h}}\left\langle\nabla u_{h N}, \nabla \tilde{\varphi_{h}}\right\rangle \text { avec }: \tilde{\varphi}_{h}=\tilde{\varphi} o F_{h}^{-1} .
$$

Par intégration par parties, on obtient :

$$
\left\langle\frac{\partial u_{N}^{h}}{\partial \nu}, \varphi\right\rangle=0
$$

Théorème 3.1. Désignons par d la distance de Haussdorff sur $\gamma$. Alors, si $\gamma_{N}^{h}$ a un nombre de composantes connexes borné par $L$ indépemndamment de $h$, on a :

$$
\lim _{h \rightarrow 0^{+}} d\left(\gamma_{N}^{h}, \gamma_{N}\right)=0
$$

Preuve. $\quad\left(\gamma_{D}, \gamma_{N}\right)$ et $\left(\gamma_{D}^{h}, \gamma_{N}^{h}\right)$ forment deux partitions de $\gamma$, il nous suffit donc de montrer, compte tenu du lemme 3.1 que $\lim _{h \rightarrow 0^{+}} \mu\left(\gamma_{N}^{h} \backslash \gamma_{N}\right)=0$.

À un difféomorphisme près on peut supposer que $\gamma=] 0,1[$ et que :

$$
\left.\gamma_{N}^{h}=\bigcup_{i=1}^{L}\right] x_{i}^{h}, y_{i}^{h}[
$$

avec :

$$
0 \leq x_{1}^{h} \leq y_{1}^{h} \leq x_{2}^{h} \leq y_{2}^{h} \leq \ldots \leq x_{L}^{h} \leq y_{L}^{h} \leq 1
$$

Supposons que la suite bornée $\mu\left(\gamma_{N}^{h} \backslash \gamma_{N}\right)$ a une valeur d'adhérence $\lambda>0$. On peut alors en extraire une sous suite encore indexée par $h$ telle que :

$$
\left\{\begin{array}{l}
1-\text { les suites } x_{i}^{h} \text { et } y_{i}^{h} \text { convergent vers } x_{i} \text { et } y_{i} \forall i \in\{1,2, \ldots, L\} \\
2-\lim _{h \rightarrow 0^{+}} \mu\left(\gamma_{N}^{h} \backslash \gamma_{N}\right)=\lambda>0
\end{array}\right.
$$

On aura alors pour un certain $i_{0} \in\{1,2, \ldots, L\}$

$$
\mu(] x_{i_{0}}, y_{i_{0}}\left[\backslash \gamma_{N}\right)>0
$$

Soit $\varphi \in D(] x_{i_{0}}, y_{i_{0}}[)$. Grâce à $(26)$, on sait qu'il existe un nombre réel $h_{0}>0$ tel que pour tout $h<$ $h_{0}$, on ait $\left.\operatorname{supp}(\varphi) \subset\right] x_{i_{0}}^{h}, y_{i_{0}}^{h}[$. 
Or, $\frac{\partial u_{N}^{h}}{\partial \nu}=0$ sur $] x_{i_{0}}^{h}, y_{i_{0}}^{h}[$. En appliquant le lemme 3.2 à $\varphi$, on obtient après passage à la limite en $h$ :

$$
\left\langle\frac{\partial u_{N}^{0}}{\partial n}, \varphi\right\rangle=0
$$

Il en résulte que $\frac{\partial u_{N}^{0}}{\partial n}=0$ sur $] x_{i_{0}}, y_{i_{0}}\left[\right.$. Sur l'ensemble (]$x_{i_{0}}, y_{i_{0}}\left[\backslash \gamma_{N}\right)$, qui est de mesure positive d'après (27), on a donc $\frac{\partial u_{N}^{0}}{\partial n}=0$. Or cet ensemble contient des ouverts, car $\gamma_{N}$ a un nombre fini de composantes connexes, et ces ouverts sont dès lors contenus dans $\gamma_{D}^{\circ}$ ce qui contredit (18).

\subsection{Dérivation de la fonction coût}

Lemme 3.4. Désignons par $u_{D}^{1}$ la dérivée lagrangienne du problème (13), si $\gamma_{N}^{h}$ a un nombre borné de composantes connexes, alors on $a$ :

$$
\int_{\Omega_{\gamma}}\left\langle\nabla u_{D}^{0}, \nabla u_{D}^{1}\right\rangle=0
$$

Preuve. On a :

$$
\int_{\Omega_{\gamma}}\left\langle\nabla u_{D}^{0}, \nabla u_{D}^{1}\right\rangle=-\int_{\Omega_{\gamma}} \Delta u_{D}^{0} u_{D}^{1}+\int_{\partial \Omega_{\gamma}} \frac{\partial u_{D}^{0}}{\partial n} u_{D}^{1} .
$$

Or, $\Delta u_{D}^{0}=0$ dans $\Omega_{\gamma}, \frac{\partial u_{D}^{0}}{\partial n}=0 \operatorname{sur} \gamma_{N} \cup\left(\Gamma_{N} \backslash \operatorname{supp}(\phi)\right)$ et $u_{D}^{1}=0 \operatorname{sur} \Gamma_{D} \cup \operatorname{supp}(\phi)$ donc :

$$
\int_{\Omega_{\gamma}}\left\langle\nabla u_{D}^{0}, \nabla u_{D}^{1}\right\rangle=\int_{\gamma_{D}} \frac{\partial u_{D}^{0}}{\partial n} u_{D}^{1}
$$

Montrons à présent que $u_{D}^{1}=0 \mathrm{pp} \operatorname{sur} \gamma_{D}$. Pour celà, soit $\mathcal{R}$ un ouvert de $\gamma$ tel que $\overline{\mathcal{R}} \subset \gamma_{D}^{\circ}$. Le théorème 3.1, nous donne l'existence d'un nombre réel $h_{0}>0$ tel que, pour tout $h<h_{0}$, on ait $\mathcal{R} \subset \gamma_{D}^{h}$. On en déduit que $u_{D}^{h}=0$ sur $\mathcal{R}$ pour tout $h<h_{0}$, et par suite que $u_{D}^{1}=0$ pp sur $\mathcal{R}$. Or $\gamma_{D}$ a un nombre borné de composantes connexes (à un ensemble négligeable près), et il en résulte que $u_{D}^{1}=0$ sur $\gamma_{D}$, ce qui achève la démonstration.

Théorème 3.2. Soit $\gamma_{h}=(I d+h \theta)(\gamma)$. Si $\gamma_{N}^{h}$ a un nombre borné de composantes connexes, alors :

$$
\lim _{h \rightarrow 0^{+}} \frac{J\left(\gamma_{h}\right)-J(\gamma)}{h}=2 \int_{\Omega_{\gamma}}\left\langle D \theta \nabla u_{N}^{0}, \nabla u_{N}^{0}\right\rangle-2 \int_{\Omega_{\gamma}}\left\langle D \theta \nabla u_{D}^{0}, \nabla u_{D}^{0}\right\rangle-\int_{\Omega_{\gamma}} \operatorname{div} \theta\left(\left|\nabla u_{N}^{0}\right|^{2}-\left|\nabla u_{D}^{0}\right|^{2}\right) .
$$

Si en outre $u_{N}^{0}$ et $u_{D}^{0} \in \mathrm{H}^{2}(\operatorname{supp}(\theta))$ on a aussi :

$$
\lim _{h \rightarrow 0^{+}} \frac{J\left(\gamma_{h}\right)-J(\gamma)}{h}=\int_{\gamma_{D}}\left[\left(\frac{\partial u_{N}^{0}}{\partial n}\right)^{2}-\left(\frac{\partial u_{D}^{0}}{\partial n}\right)^{2}\right] \theta_{n}+\int_{\gamma_{N}}\left[\left(\frac{\partial u_{D}^{0}}{\partial \tau}\right)^{2}-\left(\frac{\partial u_{N}^{0}}{\partial \tau}\right)^{2}\right] \theta_{n} .
$$

Preuve. Commençons par rappeler (voir par exemple [16]) le problème variationnel vérifié par $u_{N}^{1}$ :

$$
\left\{\begin{array}{l}
\text { Trouver } u_{N}^{1} \in \mathcal{S} \text { vérifiant, pour tout } v \in \mathcal{S}: \\
\int_{\Omega_{\gamma}}\left\langle\nabla u_{N}^{1}, \nabla\left(v-u_{N}^{1}\right)\right\rangle \geq \int_{\Omega_{\gamma}}\left\langle\left(D \theta^{t}+D \theta\right) \nabla u_{N}^{0}, \nabla\left(v-u_{N}^{1}\right)\right\rangle-\int_{\Omega_{\gamma}}\left\langle\nabla u_{N}^{0}, \nabla\left(v-u_{N}^{1}\right)\right\rangle \operatorname{div} \theta
\end{array}\right.
$$

avec :

$$
\mathcal{S}=\left\{u \in \mathrm{H}^{1}\left(\Omega_{\gamma}\right) ; u \mid \Gamma_{D}=0 ; u \geq 0 \text { p.p. sur } \gamma_{D} \text { et } \int_{\Omega_{\gamma}}\left\langle\nabla u_{N}^{0}, \nabla u\right\rangle=\int_{\Gamma_{N}} \phi u\right\} .
$$


Désignons par $J^{1}, J_{N}^{1}$ et $J_{D}^{1}$ respectivement les dérivées par rapport au domaine des fonctionnelles $J, J_{N}:=$ $\left|u_{N}^{0}\right|_{1, \Omega_{\gamma}}^{2}$ et $J_{D}:=\left|u_{D}^{0}\right|_{1, \Omega_{\gamma}}^{2}$. Alors :

$$
\int_{\Omega_{\gamma}}\left\langle\nabla u_{N}^{0}, \nabla u_{D}^{0}\right\rangle=\int_{\Gamma_{N}} \phi f
$$

Ce terme étant constant, sa dérivée par rapport au domaine est nulle et par suite $J^{1}=J_{N}^{1}+J_{D}^{1}$ avec :

$$
J_{N}^{1}=\int_{\Omega_{\gamma}} \operatorname{div} \theta\left|\nabla u_{N}^{0}\right|^{2}-2 \int_{\Omega_{\gamma}}\left\langle D \theta \nabla u_{N}^{0}, \nabla u_{N}^{0}\right\rangle+2 \int_{\Omega_{\gamma}}\left\langle\nabla u_{N}^{1}, \nabla u_{N}^{0}\right\rangle
$$

et

$$
J_{D}^{1}=\int_{\Omega_{\gamma}} \operatorname{div} \theta\left|\nabla u_{D}^{0}\right|^{2}-2 \int_{\Omega_{\gamma}}\left\langle D \theta \nabla u_{D}^{0}, \nabla u_{D}^{0}\right\rangle+2 \int_{\Omega_{\gamma}}\left\langle\nabla u_{D}^{1}, \nabla u_{D}^{0}\right\rangle .
$$

En appliquant (29) à la fonction test $v_{1}=u_{N}^{1}+u_{N}^{0}$, puis à $v_{2}=u_{N}^{1}-u_{N}^{0}$ qui appartiennent toutes deux aux convexes $S$, on déduit que :

$$
\int_{\Omega_{\gamma}}\left\langle\nabla u_{N}^{0}, \nabla u_{N}^{1}\right\rangle=2 \int_{\Omega_{\gamma}}\left\langle D \theta \nabla u_{N}^{0}, \nabla u_{N}^{0}\right\rangle-\int_{\Omega_{\gamma}} \operatorname{div} \theta\left|\nabla u_{N}^{0}\right|^{2}
$$

ce qui, avec (30), nous donne :

$$
J_{N}^{1}=2 \int_{\Omega_{\gamma}}\left\langle D \theta \nabla u_{N}^{0}, \nabla u_{N}^{0}\right\rangle-\int_{\Omega_{\gamma}} \operatorname{div} \theta\left|\nabla u_{N}^{0}\right|^{2} .
$$

Le lemme 3.4 et (31) nous donnent pour leur part :

$$
J_{D}^{1}=\int_{\Omega_{\gamma}} \operatorname{div} \theta\left|\nabla u_{D}^{0}\right|^{2}-2 \int_{\Omega_{\gamma}}\left\langle D \theta \nabla u_{D}^{0}, \nabla u_{D}^{0}\right\rangle
$$

ce qui complète la démonstration de la première partie du théorème.

La deuxième partie de ce théorème s'obtient par intégration par parties. En effet :

$$
\int_{\Omega_{\gamma}}\left\langle D \theta \nabla u_{N}^{0}, \nabla u_{N}^{0}\right\rangle=\int_{\Omega_{\gamma}} \frac{\partial u_{N}^{0}}{\partial x}\left\langle\nabla \theta_{1}, \nabla u_{N}^{0}\right\rangle+\int_{\Omega_{\gamma}} \frac{\partial u_{N}^{0}}{\partial y}\left\langle\nabla \theta_{2}, \nabla u_{N}^{0}\right\rangle
$$

ce qui, par integration par parties, nous donne :

$$
\begin{aligned}
\int_{\Omega_{\gamma}}\left\langle D \theta \nabla u_{N}^{0}, \nabla u_{N}^{0}\right\rangle= & -\int_{\Omega_{\gamma}} \theta_{1} \operatorname{div}\left(\frac{\partial u_{N}^{0}}{\partial x} \nabla u_{N}^{0}\right)+\int_{\gamma} \theta_{1} \frac{\partial u_{N}^{0}}{\partial x} \frac{\partial u_{N}^{0}}{\partial n} \\
& -\int_{\Omega_{\gamma}} \theta_{2} \operatorname{div}\left(\frac{\partial u_{N}^{0}}{\partial y} \nabla u_{N}^{0}\right)+\int_{\gamma} \theta_{2} \frac{\partial u_{N}^{0}}{\partial y} \frac{\partial u_{N}^{0}}{\partial n} .
\end{aligned}
$$

Il en résulte que :

$$
\int_{\Omega_{\gamma}}\left\langle D \theta \nabla u_{N}^{0}, \nabla u_{N}^{0}\right\rangle=-\int_{\Omega_{\gamma}} \theta_{1} \operatorname{div}\left(\frac{\partial u_{N}^{0}}{\partial x} \nabla u_{N}^{0}\right)-\int_{\Omega_{\gamma}} \theta_{2} \operatorname{div}\left(\frac{\partial u_{N}^{0}}{\partial y} \nabla u_{N}^{0}\right)+\int_{\gamma} \frac{\partial u_{N}^{0}}{\partial n}\left\langle\theta, \nabla u_{N}^{0}\right\rangle .
$$


Or, $\theta$ est porté par la normale sur $\gamma$, d'où on déduit :

$$
\int_{\Omega_{\gamma}}\left\langle D \theta \nabla u_{N}^{0}, \nabla u_{N}^{0}\right\rangle=-\int_{\Omega_{\gamma}} \theta_{1} \operatorname{div}\left(\frac{\partial u_{N}^{0}}{\partial x} \nabla u_{N}^{0}\right)-\int_{\Omega_{\gamma}} \theta_{2} \operatorname{div}\left(\frac{\partial u_{N}^{0}}{\partial y} \nabla u_{N}^{0}\right)+\int_{\gamma} \theta_{n}\left(\frac{\partial u_{N}^{0}}{\partial n}\right)^{2} .
$$

On a aussi :

$$
\int_{\Omega_{\gamma}} \operatorname{div} \theta\left|\nabla u_{N}^{0}\right|^{2}=-\int_{\Omega_{\gamma}}\left\langle\theta, \nabla\left(\left|\nabla u_{N}^{0}\right|^{2}\right)\right\rangle+\int_{\gamma} \theta_{n}\left|\nabla u_{N}^{0}\right|^{2}
$$

or :

$$
2 \operatorname{div}\left(\frac{\partial u_{N}^{0}}{\partial x} \nabla u_{N}^{0}\right)-\frac{\partial}{\partial x}\left(\left|\nabla u_{N}^{0}\right|^{2}\right)=2 \frac{\partial u_{N}^{0}}{\partial x} \Delta u_{N}^{0}
$$

et

$$
2 \operatorname{div}\left(\frac{\partial u_{N}^{0}}{\partial y} \nabla u_{N}^{0}\right)-\frac{\partial}{\partial y}\left(\left|\nabla u_{N}^{0}\right|^{2}\right)=2 \frac{\partial u_{N}^{0}}{\partial y} \Delta u_{N}^{0}
$$

Les équations (32), (34), (35), (36), et (37) nous donnent, avec l'harmonicité de $u_{N}^{0}$ :

$$
J_{N}^{1}=2 \int_{\gamma} \theta_{n}\left(\frac{\partial u_{N}^{0}}{\partial n}\right)^{2}-\int_{\gamma} \theta_{n}\left|\nabla u_{N}^{0}\right|^{2}
$$

et encore :

$$
J_{N}^{1}=\int_{\gamma_{D}} \theta_{n}\left(\frac{\partial u_{N}^{0}}{\partial n}\right)^{2}-\int_{\gamma_{N}}\left(\frac{\partial u_{N}^{0}}{\partial \tau}\right)^{2}
$$

De même on montre que :

$$
J_{D}^{1}=-\int_{\gamma_{D}} \theta_{n}\left(\frac{\partial u_{D}^{0}}{\partial n}\right)^{2}+\int_{\gamma_{N}}\left(\frac{\partial u_{D}^{0}}{\partial \tau}\right)^{2}
$$

\subsection{Remarques}

1. Essentielle pour la démonstration du théorème, l'hypothèse sur le nombre de composantes connexes de $\gamma_{N}^{h}$ est à notre connaissance invérifiable. Bien plus, il est même tout à fait possible de construire des solutions du problème de Signorini qui la violent, au prix il est vrai de fortes oscillations dont il semble pas très restrictif de supposer que la solution réelle s'abstient. Nous noterons par ailleurs l'hypothèse en question s'est déjà imposée à d'autres auteurs traitant de problèmes avec conditions aux limites de Signorini [3,4].

2. Si on impose un flux $\phi \geq 0$ sur $\Gamma_{N}$, le principe du maximum nous permet de déduire que la condition Signorini sur $\gamma$ se réduit à une condition de type Neumann. En effet, le minimum de $u_{N}$ ne peut être atteint ni sur $\Gamma_{N}$, ni sur $\gamma$ puisque $\frac{\partial u_{N}^{0}}{\partial n}<0$ en tout point de la frontière où ce minimum est atteint. En conséquence, le minimum est atteint sur $\Gamma_{D}$, et il est donc nul. On en conclut que $\gamma_{D}$ est vide faute de quoi le minimum serait également atteint sur $\gamma_{D}$ donc sur $\gamma$. L'expression du gradient de la fonctionnelle $J$ devient :

$$
J^{1}=\int_{\gamma_{N}}\left[\left(\frac{\partial u_{D}^{0}}{\partial \tau}\right)^{2}-\left(\frac{\partial u_{N}^{0}}{\partial \tau}\right)^{2}\right] \theta_{n}
$$


c'est-à-dire que l'on retrouve des résultats déjà démontrés dans le cas linéaire [1].

3. Si on applique un flux $\phi \leq 0$ sur $\Gamma_{N}$, le principe du maximum nous permet de conclure de la même façon que le maximum $u_{M}$ de $u_{N}^{0}$ ne peut être atteint en un point de $\Gamma_{N}$ car on a $\frac{\partial u_{N}^{0}}{\partial n}>0$ en tout point de la frontière où le maximum est atteint. Ce maximum est donc nul, puisqu'il est atteint soit en un point de $\Gamma_{D}$, soit en un point de $\dot{\gamma}_{D}^{\circ}$. Il en résulte que $\gamma_{N}$ est vide car sinon ce maximum ne serait pas nul. La condition de Signorini sur $\gamma$ se réduit alors à une condition de type Dirichlet $u_{N}^{0}=0$ sur $\gamma$. Dans ce cas, $\mu\left(\gamma_{N}\right)=0$, et le théorème 3.2 nous donne :

$$
J^{1}=\int_{\gamma_{D}}\left[\left(\frac{\partial u_{N}^{0}}{\partial n}\right)^{2}-\left(\frac{\partial u_{D}^{0}}{\partial n}\right)^{2}\right] \theta_{n}
$$

c'est-à-dire que l'on retrouve les résultats de J.R. Roche et J. Sokolowski [14] pour le cas linéaire.

\section{Mise en ceuvre numÉRIQue et CONClusions}

Ce paragraphe a pour objet d'illustrer la faisabilité et la validité de l'algorithme proposé, dont l'évaluation des performances est cependant loin d'être exhaustive à ce stade. Les calculs ont été effectués pour un domaine carré dont le quatrième «côté» est la frontière inconnue $\gamma$. Celle-ci est paramétrée par un nombre $N$ d'inconnues représentant les ordonnées des points situés aux abcisses $x_{i}=\frac{i}{N} ; i=1, N$. Les extrémités fixes de la frontière $\gamma$ sont les points $(0,1)$ et $(1,1)$.

La fonction coût que nous avons définie, et les deux expressions calculées de sa dérivée directionnelle par rapport au domaine, permettent la mise en œuvre, pour la résolution numérique du probème inverse de Signorini, d'un algorithme de gradient à pas constant fonctionnant de la manière suivante :

1. Étant donnée une géométrie de la frontière $\gamma$, le maillage du domaine correspondant $\Omega_{\gamma}$ et le calcul des matrices de rigidité correspondant à la discrétisation par éléments finis $P_{1}$ des deux problèmes directs (8) et (13), sont effectués en faisant appel aux modules de la bibliothèque Modulef, en aval de laquelle nous avons programmé les solveurs que nous avons utilisés. Le premier problème, non linéaire, a été résolu par la méthode du gradient avec projection, particulièrement aisée à mettre en œuvre dans le cadre d'une approximation par éléments finis $P_{1}$. Cette première étape nous donne les solutions $u_{D}^{0}$ et $u_{N}^{0}$ correspondant à la frontière d'initialisation.

2. On calcule le gradient de la fonction coût grâce aux expressions données dans le théorème 3.2. Pour le problème discrétisé, ce gradient comporte autant de composantes que de directions virtuelles de déformation de la frontière inconnue. Dans tous les cas, le calcul du gradient ne fait appel à aucune résolution, seulement à des calculs d'intégrales faisant intervenir les solutions $u_{D}^{0}$ et $u_{N}^{0}$ déjà calculées.

3. La frontière $\gamma$ est réactualisée dans la direction du gradient calculé ci-dessus, avec un pas constant $\rho$. Si le test d'arrêt - portant sur la valeur de la fonction coût - n'est pas satisfait, on repart en 1.

À chaque itération de modification de la géométrie, le domaine est entièment remaillé, et les matrices de rigidité des deux problèmes directs (13) et (8) intervenant dans la définition de la fonction coût recalculées. Dans cette mise en œuvre, nous avons rencontré un certain nombre de problèmes auxquels les réponses apportées - d'ordre plus numérique que théorique - ont néanmoins permis d'obtenir des résultats plus satisfaisants :

1. Il est apparu qu'une représentation fine des variations de la frontière inconnue $\gamma$ dès les premières itérations de l'algorithme nuit à la qualité de sa convergence. Cette difficulté est surmontée par l'adoption d'une démarche «hiérarchique», que l'on peut décrire de la manière suivante :

(a) On fixe un nombre $N$ «petit» de directions virtuelles de variation de la géométrie inconnue, et on calcule par la méthode du gradient la «meilleure géométrie» possible dans ce cadre, c'est à dire celle qui rend minimale la fonction coût définie ; 
(b) Lorsque l'algorithme a convergé pour ce nombre $N$, on enrichit les possibilités de déformation de la géométrie, par exemple en doublant le nombre de ses directions virtuelles, l'initialisation étant évidemment effectuée avec la solution atteinte à l'étape précédente.

La figure 4, qui donne l'évolution des erreurs relatives en normes $\mathrm{L}^{2}$ et $\mathrm{L}^{\infty}$, illustre bien les différents paliers de convergence, correspondant aux étapes hiérarchiques dans la description de la frontière.

2. Nous avons choisi de paramétrer les directions virtuelles de déformation de la géométrie par les fonctions de forme de l'approximation numérique par éléments finis, mais ce choix n'est ni le seul possible, ni même vraisemblablement le meilleur. De même, nous avons déjà fait observer plus haut qu'il n'est pas souhaitable d'explorer dans un premier temps toutes les possibilités de déformation virtuelles offertes par ces fonctions de forme.

3. Une reconstruction brute de la frontière à partir des points calculés conduit à des résultats peu satisfaisants quant à la précision, et surtout à des problèmes de stabilité de l'algorithme. Une procédure de lissage est apparue indispensable pour stabiliser l'algorithme, les frontières $\gamma$ calculées après déformation sans lissage ne présentant plus la régularité requise dans [2] pour assurer le résultat de stabilité locale Lipschitzienne.

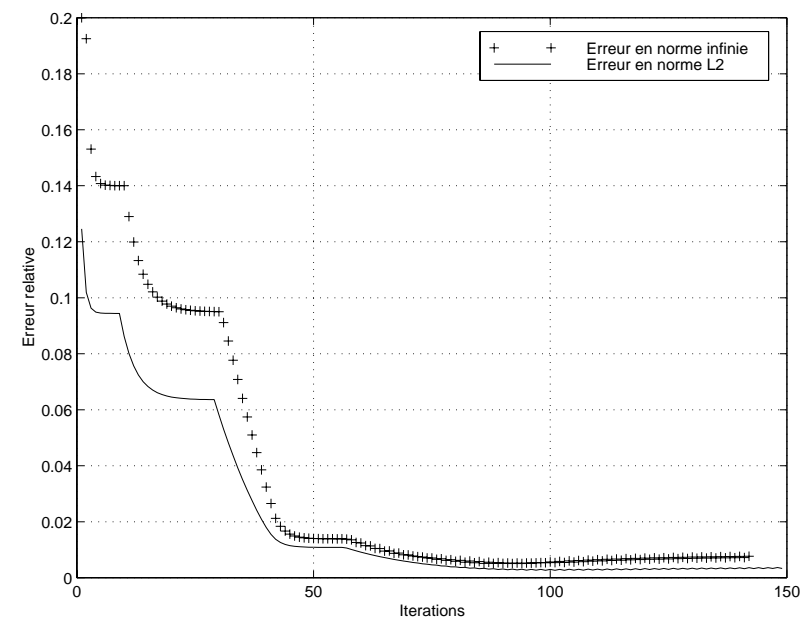

FiguRE 4. Erreur relative sur la frontière.

La linéarisation du second problème intervenant dans l'expression de la fonctionnelle $J$ contribue doublement à la diminution des coûts de calcul : d'une part, chaque itération de l'algorithme requiert la résolution d'un problème linéaire et d'un problème non linéaire (au lieu de deux problèmes non linéaires), d'autre part l'expression du gradient ne fait intervenir que les états déjà calculés, et non leurs dérivées.

Les deux figures ci-après donnent les résultats obtenus avec une méthode non hiérarchique utilisant un petit nombre de paramètres de description de la frontière sans lissage.

Avec ou sans lissage cependant, l'algorithme éprouve certaines difficultés pour suivre les changements de courbure de la frontière recherchée, comme le montrent les figures 5 et 6 , les résultats de cette dernière étant obtenus par la procédure hiérarchique décrite plus haut, avec un lissage relativement frustre (la valeur prise en chaque point est corrigée par celles prises aux deux points voisins).

En l'état actuel des choses, le nombre d'itérations reste encore assez important, et les temps de calcul relativement élevés. Des améliorations considérables pourront à l'évidence être apportées sur ce point, en envisageant de résoudre les problèmes directs pour le calcul de $u_{N}^{0}$ et $u_{D}^{0}$ par une méthode de décomposition de domaines 

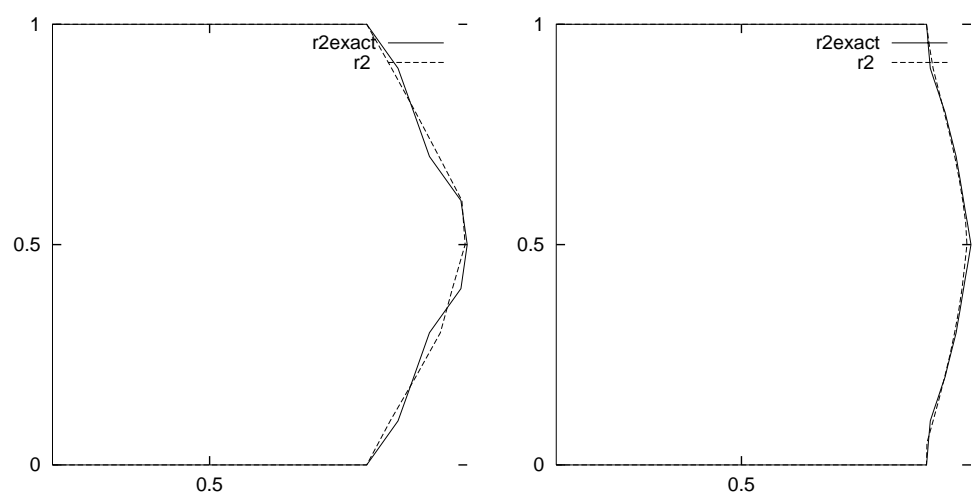

FiguRE 5. Reconstitution sans lissage de frontières inconnues.
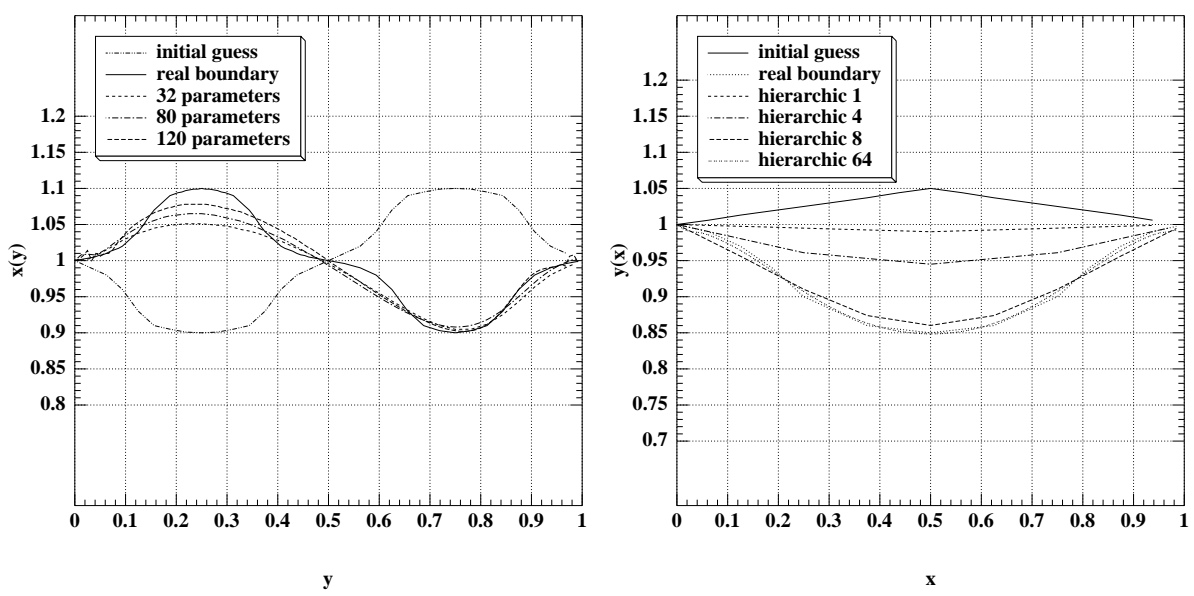

FiguRE 6. Reconstitution avec lissage de frontières inconnues.

avec maillages non conformes, de manière à pouvoir suivre avec précision les variations de la géométrie inconnue. Cette démarche, qui permettrait de ne remailler que la partie du domaine voisine de la frontière inconnue, et de ne résoudre ainsi à chaque itération de l'algorithme du gradient que des problèmes de taille réduite, n'a cependant pas été mise en œuvre pour l'obtention des résultats numériques présentés ci-dessus. Ceci étant, plus que dans les performances de l'algorithme lui-même, l'intérêt majeur du présent travail nous paraît résider dans la manière de traiter les difficultés liées à la partition de la frontière de Signorini. Cette méthode semble en effet susceptible d'être étendue au problème inverse thermoélastique couplé, qui modélise une situation physiquement plus réaliste.

Acknowledgements. Ce travail a été en partie effectué alors que le second auteur était professeur associé à la division de mathématiques appliquées de l'université de techonologie de Compiègne, qu'il tient à remercier pour son accueil. Le travail scientifique des chercheurs du LAMsIN bénéficie du soutien financier du secrétariat d'État à la recherche scientifique et à la technologie, dans le cadre du programme LAB-STI-02.

Les auteurs tiennent à remercier tout particulièrement les rapporteurs pour avoir attiré leur attention sur plusieurs points clés, notamment dans les démonstrations des Lemmes 1 et 2 . 


\section{RÉFÉRENCES}

[1] S. Andrieux, A. Ben Abda et M. Jaoua, Identifiabilité de frontiètres inaccessibles par une mesure unique de surface. Annales Maghrébines de l'Ingénieur, 7 (1993) 5-24.

[2] A. Ben Abda, S. Chaabane, F. El Dabaghi et M. Jaoua, On a non linear geometrical inverse problem of Signorini type: identifiability and stability. Math. Meth. in the Appl. Sci. 21 (1998) 1379-1398.

[3] F. Ben Belgacem, Numerical simulation of some variational inequalities arisen from unilateral contact problems by the finite element method. Sinum (à paraître).

[4] F. Brezzi, W.W. Hager et P.A. Raviart Error estimates for the finite element solution of variational inequalities. Numer. Math. 28 (1977) 431-443.

[5] S. Chaabane et M. Jaoua, Identification of Robin coefficients by the means of boundary measurements. Inverse Problems 15 (1999) 1425-1438.

[6] F. Hettlich et W. Rundell Iterative methods for the reconstraction of an inverse potential problem. Inverse Problems 12 (1996) $251-266$.

[7] K. Khodja et M. Moussaoui, Régularité des solutions d'un problème mêlé Dirichlet-Signorini dans un domaine polygonal plan. Comm. Partial Diff. Eq. 17 (1992) 805-826.

[8] R.V. Kohn et A. McKenney Numerical implementation of a variational method for electrical impedance tomography. Inverse Problems 6 (1990) 389-414.

[9] R.V. Kohn et M. Vogelius, Determinig conductivity by boundary measurements; interior results. Comm. Pure Appl. Math. 38 (1985) 644-667.

[10] R.V. Kohn et M. Vogelius, Relaxation of a variational method for impedance computed tomography. Comm. Pure Appl. Math. 40 (1987) 745-777.

[11] K. Kunisch et X. Pan, Estimation of interfaces from boundary measurements. SIAM J. Cont. Opt. 32 (1994) 867-894.

[12] J.L.M. Lions, Quelques méthodes de résolution de problèmes aux limites non linéaires. Dunod, Paris (1969).

[13] J.L. Lions et E. Magenes, Problèmes aux limites non homogènes et applications, tome 1. Dunod, Paris (1968).

[14] J.R. Roche et J. Sokolowski, Numerical methods for shape identification problems. Control and Cybernetics 25 (1996) $867-894$.

[15] J. Simon, Differentiation with respect to the domaine in boundary value problems. Num. Func. Anal. Opt. 2 (1980) 649-687.

[16] J. Sokolowski et J.P. Zolesio, Introduction to shape optimization; shape sensitivity analysis. Springer Verlag (1992). 